\title{
Cross border, highly individualised treatment of a patient with challenging extensively drug-resistant tuberculosis
}

\author{
To the Editor:
}

Extensively drug-resistant (XDR) tuberculosis (TB) is defined by resistance to isoniazid, rifampicin, any fluoroquinolone and at least one of the three second line injectable drugs, such as amikacin. Drug toxicity and duration impair adherence to treatment and outcome is rather poor [1]. We report on a particularly challenging XDR-TB patient with persistent non-adherence to treatment and an exceptionally complex drug susceptibility pattern.

On July 7, 2015, a 28-year-old British citizen with XDR-TB was transferred by air ambulance from the UK to the TB centre "Beatrixoord" in Haren, the Netherlands. He had no other relevant comorbidities and negative tests for HIV and chronic hepatitis. His father had been initially diagnosed with drug-susceptible TB in 1999 and succumbed to multidrug-resistant (MDR) TB in 2005. Despite prophylactic treatment with isoniazid during 2000, the patient was diagnosed with drug-susceptible TB in November 2007, when he started first-line TB treatment. Over the course of more than 7 years, many hospital admissions followed, while his Mycobacterium tuberculosis isolates became increasingly drug resistant and his treatment regimen ever expanded (figure 1a). Despite frequent molecular and phenotypic resistance testing, followed even by whole genome sequencing (WGS), the results for several drugs were ambiguous. By the end of 2009 his TB was classified as extensively drug resistant. Two consecutive negative cultures were obtained mid-2011 and again in 2012, but both times this was followed by culture reversion (figure 1a). Our patient was addicted to cannabis and had many social problems resulting in poor adherence to therapy. Other possible causes of a failing regimen, like poor drug absorption, were considered, but at that time it was believed that the extremely poor adherence was the major problem. In mid-2015, his medical team felt that only a highly individualised treatment regimen focusing not only on pharmacotherapy, but also on a change in social environment and social habits might curb impending fatal outcome. This was difficult to realise in the UK, because the Public Health Act lacks a compulsory treatment order and sanatoria are not available. The TB centre in Groningen, the Netherlands provides an environment conducive to long-term treatment of socially complex and medically challenging TB cases. In addition, the Dutch Public Health Act enables long-term mandatory isolation of patients unwilling to follow infection control procedures. Our TB centre and the Dutch national health authorities were approached by the National Health Service (NHS) to discuss the patient's transfer. The health authorities of both countries supported the transfer and the NHS and our TB centre agreed on the conditions, including financial arrangements, and the patient gave his consent.

In the first months thereafter, the National Institute for Public Health and the Environment (RIVM, Bilthoven, the Netherlands) performed drug susceptibility testing (DST) on current and previous M. tuberculosis sputum isolates. Based on the first test results we started treatment with high dose rifampicin, isoniazid, ethambutol, cycloserine and linezolid [2]. Therapeutic drug monitoring (TDM) was performed to optimise treatment [3]. Further analysis revealed the existence of several minority populations of $M$. tuberculosis with ambiguous susceptibilities to rifampicin and amikacin. Consistent resistance profiles were, however, obtained after isolation of colonies from $7 \mathrm{H} 10$ agar with the highest concentrations of these two drugs ( 2 and $5 \mathrm{mg} \cdot \mathrm{L}^{-1}$ respectively) [4]. Of these isolated colonies, molecular targets (rpoB (ATC 491 TTC $\rightarrow$ TAC) and $r r s$ gene $(A 1401 \rightarrow \mathrm{G})$ ) as well as the full drug susceptibility profiles for all drugs were determined. The highly rifampicin and amikacin resistant minority populations,

@ERSpublications

Crossing borders by treating a patient with difficult to treat XDR-TB; highly individualised but holistic approach http://ow.ly/JyjK30ielsD

Cite this article as: Akkerman OW, Grasmeijer F, de Lange WCM, et al. Cross border, highly individualised treatment of a patient with challenging extensively drug-resistant tuberculosis. Eur Respir $J$ 2018; 51: 1702490 [https://doi.org/10.1183/13993003.02490-2017]. 
a)

rpoB 1491F\#
embB M306

Kanamycin

Moxifloxacin

Ofloxacin

Ciprofloxacin

Amikacin

Meropenem

Amoxicillin/clavulanate

Linezolid

Clofazimine

Clarithromycin

Protionamide

Cycloserine

Para-aminosalicylic acid

Capreomycin

Ethambutol

Pyrazinamide

Isoniazid

Rifampicin

Culture

Smear

1-1-2007

i
pncA V125G

katG V473L pncA V7L?

,
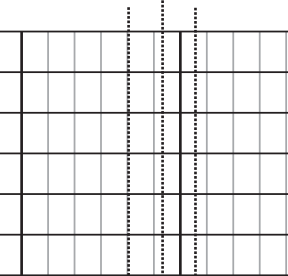

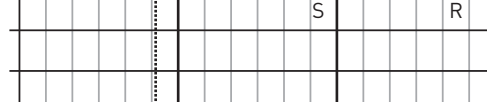

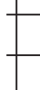
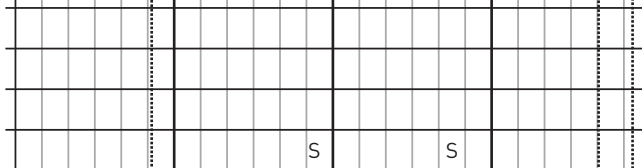

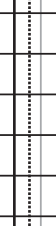

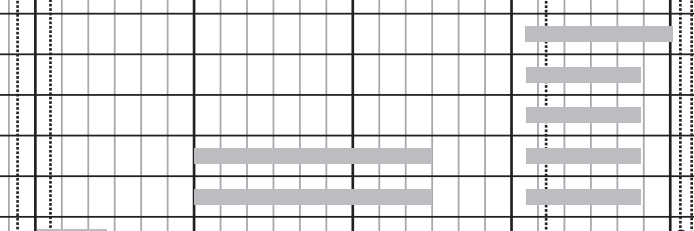

fabG1C-15T rrsA1401Nף rpoB $1491 \mathrm{~F} / \mathrm{Y} \S$ :

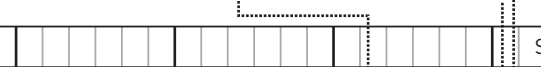

S

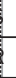
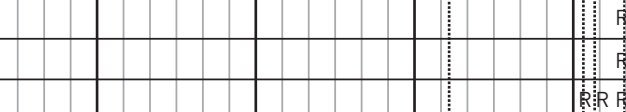

के $s$
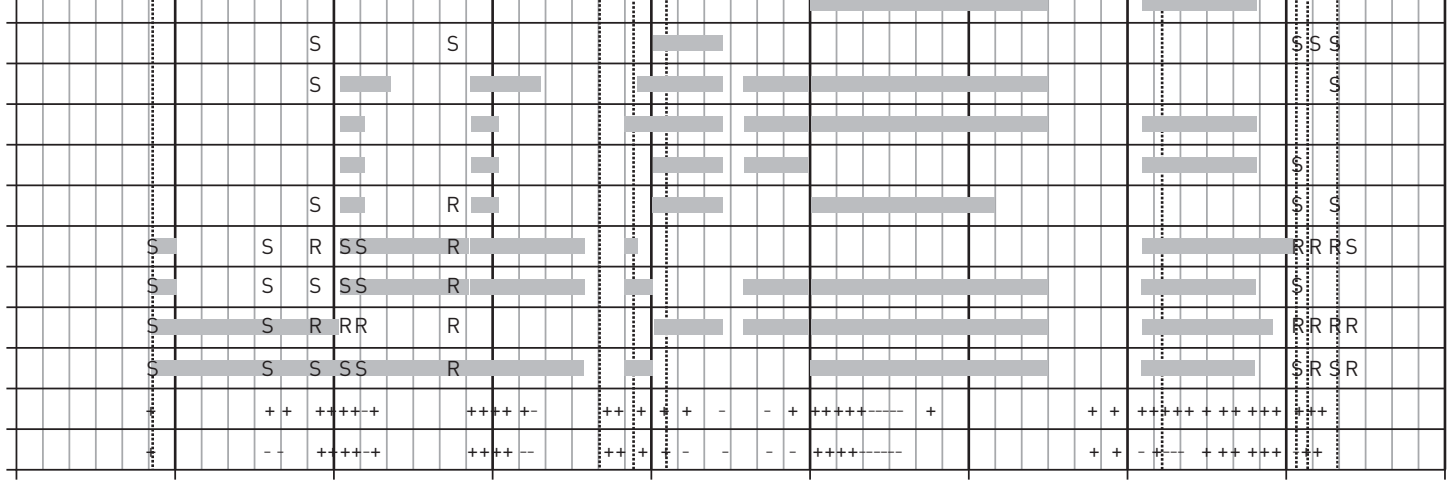

b)

Start individualised treatment

Discharge and start DOT

End of treatment: Patient cured

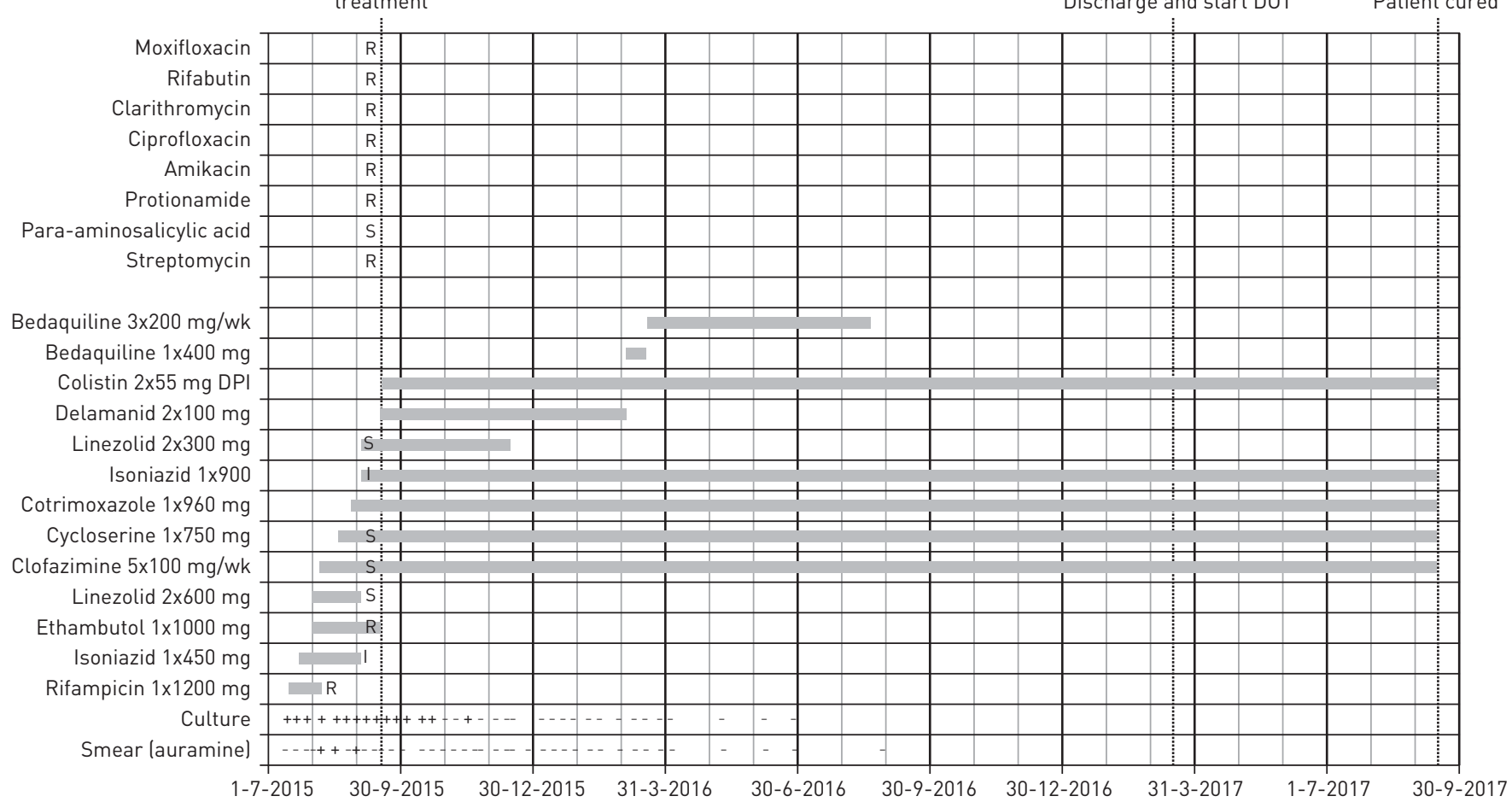

FIGURE 1 Timeline including the pharmaco-therapeutic treatment, sputum test results and results of drug susceptibility testing for the treatment in a) the UK and b) the Netherlands. " : disputed mutation encoding low level rifampicin resistance; " : mixed wild-type/mutant; ${ }^{\S}$ : mixed second mutation in the rpoB gene encoding high level rifampicin resistance found after isolation of colonies on agar containing a high concentration of rifampicin or amikacin. S: susceptible; R: resistant; I: intermediate resistant; +: positive; -: negative; DPI: dry powder inhalation; DOT: directly observed therapy. 
initially remaining undetected by WGS, had a similar resistance profile. Molecular analysis of the resistance profiles showed the highly resistant minority populations in later cultured strains without drug selection, indicating that their numbers increased over time. Rifampicin was discontinued since a high cumulative minimal inhibitory concentration was found for this drug. Based on an earlier study [5], $960 \mathrm{mg}$ co-trimoxazole once daily was added to the regimen. Furthermore, the isoniazid dose was increased to $900 \mathrm{mg}$. With only few effective drugs available according to DST results, we added delamanid and bedaquiline in consecutive order. For earlier culture conversion we considered adding yet another drug. Thiacetazone is poorly available and we therefore opted for inhaled colistin. In vitro studies have demonstrated an isoniazid-potentiating effect of colistin $[6,7]$, and it was shown that the required colistin concentrations in sputum to achieve such effects could be reached by dry powder inhalation $[6,8]$. We used the Twincer ${ }^{\circ}$ dry powder inhaler (PureIMS, Roden, the Netherlands) to minimise adverse reactions such as cough $[8,9]$. We instructed correct inhalation for efficacy. Adverse drug reactions were only observed for linezolid, which was discontinued after the patient started to develop peripheral neuropathy. Figure $1 \mathrm{~b}$ provides an overview of the pharmaco-therapeutic regimen given. Surgical resection as a complementary treatment was deemed technically unfeasible, because of the bilateral involvement, the insufficient pulmonary reserve and the extensive scarring.

In addition to a change in pharmaco-therapeutic treatment the Dutch $\mathrm{TB}$ centre provided a change in physical and social environment that helped our patient to adhere to treatment. Not only was he detached from his complex social life, but we could also offer him several social and physical daily activities tailored to his personal interests and abilities.

With the support of the nursing staff, he managed to adhere to therapy and within 2 months of the start of his tailor-made drug treatment, his sputum smear and culture converted negative (figure 1b). After 18 months of hospitalisation, he continued his treatment as an outpatient in Groningen, the Netherlands with directly observed therapy 5 days per week in our TB centre. Treatment was considered completed by 24 months. Bilateral radiological abnormalities in the upper lung lobes have persisted without any complications.

To achieve success, several borders were crossed. First, moving the patient far away from his social environment that precluded treatment adherence was crucial. Second, his transfer to the TB centre enabled him to follow an individualised social programme in a hospitable physical environment that helped him to comply with treatment. Third, the problem of highly complicated molecular and phenotypic DST results was solved by isolating the most resistant bacteria on DST agar plates. All relevant resistance profiles of the causative bacteria were disclosed and therapy could be tailored and adjusted accordingly. Despite using cutting edge technology including WGS, initial DST predictions failed because of minority bacterial populations hidden by abundantly present susceptible bacteria. We speculate that highly resistant minority bacterial populations may be critical in patients with a long treatment history. Fourth, TDM helped to optimise treatment with the limited pharmaco-therapeutic options available. We targeted drug concentrations within the therapeutic window, thereby reducing toxicity and increasing the chance of a favourable outcome [10].

Finally, we were able to expand our regimen with several experimental drugs. Apart from adding delamanid, bedaquiline and co-trimoxazole, a drug with a reasonable safety and efficacy profile [5], we were the first to apply dry powder inhalation of colistin for the potentiation of high dose isoniazid in this patient. It has been hypothesised that hydrophilic antibiotics in general could benefit from this potentiating effect of colistin [6], and therefore, cycloserine $(\log \mathrm{P}=-0.9)$ may have been potentiated as well.

Close collaboration between all stakeholders in the treatment of MDR- and XDR-TB in the Netherlands has resulted in a treatment success rate as high as that of drug-susceptible TB [11, 12]. Most patients coming to the TB departments of Dutch municipal health services are foreign-born; the TB unit has received patients with different language and cultural backgrounds. National programmes already work together across borders, but they might also consider sharing highly specialised well-resourced facilities to treat highly complex TB cases.

Onno W. Akkerman ${ }^{1,2,13}$, Floris Grasmeijer ${ }^{3,13}$, Wiel C.M. de Lange ${ }^{1,2}$, Huib A.M. Kerstjens ${ }^{1}$, Gerard de Vries ${ }^{4,5}$, Mathieu S. Bolhuis ${ }^{6}$, Jan-Willem Alffenaar ${ }^{6}$, Henderik W. Frijlink ${ }^{3}$, Grace Smith ${ }^{7}$, Roger Gajraj ${ }^{8}$, Rina de Zwaan', Paul Hagedoorn ${ }^{3}$, Martin Dedicoat ${ }^{10}$, Dick van Soolingen ${ }^{9,11}$ and Tjip S. van der Werf ${ }^{1,12}$

${ }^{1}$ University of Groningen, University Medical Center Groningen, Dept of Pulmonary Diseases and Tuberculosis, Groningen, The Netherlands. ${ }^{2}$ University of Groningen, University Medical Center Groningen, Tuberculosis Center Beatrixoord, Haren, The Netherlands. ${ }^{3}$ University of Groningen, Dept of Pharmaceutical Technology and Biopharmacy, Groningen, The Netherlands. ${ }^{4} \mathrm{KNCV}$ Tuberculosis Foundation, Den Haag, The Netherlands. ${ }^{5}$ Centre for Infectious Disease Control, National Institute for Public Health and the Environment (RIVM), Bilthoven, The Netherlands. ${ }^{6}$ University of Groningen, University Medical Center Groningen, Dept of Clinical Pharmacy and Pharmacology, 
Groningen, The Netherlands. ${ }^{7}$ National Mycobacteriology Reference Unit, Birmingham, UK. ${ }^{8}$ Public Health England, Birmingham, UK. ${ }^{9}$ National Tuberculosis Reference Laboratory, National Institute for Public Health and the Environment (RIVM), Bilthoven, The Netherlands. ${ }^{10}$ Dept of Infection, Heart of England Foundation Trust, Birmingham, UK. ${ }^{11}$ Radboud University Nijmegen Medical Centre, Depts of Pulmonary Diseases and Medical Microbiology, Nijmegen, The Netherlands. ${ }^{12}$ University of Groningen, University Medical Center Groningen, Dept of Internal Medicine, Groningen, The Netherlands. ${ }^{13}$ Both authors contributed equally.

Correspondence: Onno W. Akkerman, University Medical Center Groningen, Tuberculosis Center Beatrixoord, Dilgtweg 5, 9751 ND Haren, Groningen, The Netherlands. E-mail: o.w.akkerman@umcg.nl

Received: Dec 012017 | Accepted after revision: Jan 202018

Conflict of interest: F. Grasmeijer reports part time employment by PureIMS BV, outside the submitted work. H.W. Frijlink has a patent for a breath actuated dry powder inhaler (number WO 2015/187025 A1) pending. P. Hagedoorn has a patent pending and is co-inventor of Twincer. T.S. van der Werf was principal investigator of the RUTI therapeutic vaccine trial, sponsored in part by Archivel, Badelona, Spain; and participated in a meeting organised and sponsored by TBVI in June, 2016, and in a meeting organised by TBVI and sponsored by Transgene-Institut Merieux France, in November 2017.

\section{References}

1 Migliori GB, Sotgiu G, Gandhi NR, et al. Drug resistance beyond extensively drug-resistant tuberculosis: individual patient data meta-analysis. Eur Respir J 2013; 42: 169-179.

2 Sotgiu G, Centis R, D'Ambrosio L, et al. Linezolid to treat extensively drug-resistant TB: retrospective data are confirmed by experimental evidence. Eur Respir J 2013; 42: 288-290.

3 Ghimire S, Bolhuis MS, Sturkenboom MGG, et al. Incorporating therapeutic drug monitoring into the World Health Organization hierarchy of tuberculosis diagnostics. Eur Respir J 2016; 47: 1867-1869.

4 van Klingeren B, Dessens-Kroon M, van der Laan T, et al. Drug susceptibility testing of Mycobacterium tuberculosis complex by use of a high-throughput, reproducible, absolute concentration method. J Clin Microbiol 2007; 45: 2662-2668.

5 Alsaad N, van Altena R, Pranger AD, et al. Evaluation of co-trimoxazole in the treatment of multidrug-resistant tuberculosis. Eur Respir J 2013; 42: 504-512.

6 Bax HI, de Steenwinkel JEM, ten Kate MT, et al. Colistin as a potentiator of anti-TB drug activity against Mycobacterium tuberculosis. J Antimicrob Chemother 2015; 70: 2828-2837.

7 van Breda SV, Buys A, Apostolides Z, et al. The antimicrobial effect of colistin methanesulfonate on Mycobacterium tuberculosis in vitro. Tuberculosis 2015; 95: 440-446.

8 Schuster A, Haliburn C, Döring G, et al. Safety, efficacy and convenience of colistimethate sodium dry powder for inhalation (Colobreathe DPI) in patients with cystic fibrosis: a randomised study. Thorax 2013; 68: 344-350.

9 Hagedoorn P, Grasmeijer F, Hoppentocht M, et al. In vitro evaluation of the Twincer colistin dry powder inhaler as a non-cough-inducing alternative to Colobreathe. Eur Respir J 2016; 48: Suppl. 60, PA2561.

10 van Altena R, Dijkstra JA, van der Meer ME, et al. Reduced chance of hearing loss associated with therapeutic drug monitoring of aminoglycosides in the treatment of multidrug-resistant tuberculosis. Antimicrob Agents Chemother 2017; 61: e01400-16.

11 van Altena R, de Vries G, Haar CH, et al. Highly successful treatment outcome of multidrug-resistant tuberculosis in the Netherlands, 2000-2009. Int J Tuberc Lung Dis 2015; 19: 406-412.

12 van Altena R, Akkerman OW, Alffenaar J-WC, et al. Shorter treatment for multidrug-resistant tuberculosis: the good, the bad and the ugly. Eur Respir J 2016; 48: 1800-1802. 